\title{
Analysis of drugs of abuse in wastewater from two Canadian cities
}

\author{
Viviane Yargeau $^{\text {a,* }}{ }$, Bryanne Taylor $^{\mathrm{b}}$, Hongxia Li $^{\mathrm{b}}$, Angela Rodayan $^{\mathrm{a}}$, Chris D. Metcalfe ${ }^{\mathrm{b}}$ \\ a Department of Chemical Engineering, McGill University, Montreal, QC, Canada \\ ${ }^{\mathrm{b}}$ Water Quality Centre, Trent University, Peterborough, ON, Canada
}

\section{H I G H L I G H T S}

- One of only a few reports on community use of opioid drugs; first for North America.

- Evidence of high oxycodone use and consumption of ketamine is found in selected communities.

- Sampling rates for passive samplers are determined for the first time for 7 drugs.

- POCIS is useful for monitoring in treated WW, but has limited applicability in untreated WW.

\section{A R T I C L E I N F O}

Article history:

Received 10 September 2013

Received in revised form 16 November 2013

Accepted 17 November 2013

Available online 8 December 2013

Keywords:

Illicit drugs

Cocaine

Amphetamines

Opioids

Wastewater

\begin{abstract}
A B S T R A C T
Several drugs of abuse, including amphetamines, cocaine and its metabolite, benzoylecgonine and several opioid prescription drugs were detected in wastewater from two Canadian cities, a small community (75,000 population) and a large urban center (1.6 million population). The objective of this study was to evaluate community use of these drugs in two cities with large differences in population size and demographics. In addition, we evaluated the use of the Polar Organic Chemical Integrative Sampler (POCIS) as a monitoring tool for drugs of abuse. Heroin was not detected at either location, probably because this illicit drug is metabolized to morphine prior to excretion. Acetylcodeine and acetylmorphine were also not detected. Estimates of community consumption from wastewater analysis indicated that the most widely used drug was cocaine at a median level of consumption in the larger city of approximately 38 doses per day per 1000 people. Consumption of the substituted amphetamine, ephedrine, as well as methamphetamine was also higher in the larger city, at 21 and 1.8 doses per day per 1000 people, respectively. Use of amphetamine, MDMA and tramadol were similar in both centers, but use of oxycodone was greater in the smaller city. Use of MDMA (ecstasy) peaked on weekends. Ketamine was detected in wastewater from the larger city; the first report of abuse of this veterinary anesthetic in a North American city. POCIS sampling rates were determined for the first time for 7 of the target compounds. Comparing the time weighted average concentrations estimated from POCIS data to the concentrations obtained from 24-h composite samples, the data were generally comparable, except for some compounds which were not detected in POCIS deployed in the untreated wastewater, probably because of biofouling or accumulation of debris on the cages containing the POCIS. This study indicates that the size and demographics of population centers can influence the patterns of abuse of drugs.
\end{abstract}

(c) 2013 Elsevier B.V. All rights reserved.

\section{Introduction}

Community consumption patterns for drugs of abuse have been estimated by analyzing the concentrations of these drugs in municipal wastewater (Postigo et al., 2011; van Nuijs et al., 2011a). Our previous studies using this approach showed that community consumption patterns for cocaine and amphetamines in three Canadian cities were similar to consumption patterns in European cities, and amphetamine use was greater in larger urban centers than in small rural communities (Metcalfe et al., 2010). Opioids are a class of drugs prescribed to treat

\footnotetext{
* Corresponding author at: 3610 University, Montreal, Quebec H3A2B2, Canada. Tel.: +15143982273 .

E-mail address: Viviane.yargeau@mcgill.ca (V. Yargeau).
}

chronic or postoperative pain, although some opioids such as heroin, are recognized illicit drugs. Several prescription opioid drugs are also used illegally as drugs of abuse. The abuse of prescription opioids has become a major problem among high school students in the province of Ontario, Canada (Paglia-Boak et al., 2011). Therefore, a goal of this study was to estimate the consumption patterns for opioid drugs, as well as cocaine and amphetamines in two Canadian cities with widely different population sizes and demographics.

Significant advances have been made for using analysis of wastewater as a tool for estimating community consumption of drugs of abuse, but there are still many uncertainties associated with this technique, including a lack of understanding of the fate of drugs and their metabolites in sewers and variations in drug excretion profiles as a result of disease, sex, age and lifestyle (van Nuijs et al., 2011a). Another uncertainty is the 
choice of sampling regime for wastewater, which may bias the estimates of drug consumption. Selecting water sampling techniques and a sampling regime that accounts for variations in wastewater flows and concentrations over time is of great importance for obtaining reliable estimates of drug consumption (Ort et al., 2010; Lai et al., 2011). Previous studies on drugs of abuse, opioid prescription pharmaceuticals in sewage were based on grab samples or 24-h composite samples of untreated wastewater (Bones et al., 2007; Postigo et al., 2008).

A passive sampling technique using the Polar Organic Chemical Integrative Sampler (POCIS) has potential for use in monitoring for drugs of abuse and prescription opioids in wastewater. POCIS provides estimates of the time-weighted average concentration of compounds accumulated from aqueous matrices into a solid sorbent over the time of deployment (Jones-Lepp et al., 2004; Li et al., 2010a; Harman et al., 2011). This method may provide more accurate estimates of the average concentrations of drugs in wastewater over time than estimates obtained from grab or even 24-h composite samples. However, in order to estimate concentrations of drugs in aqueous matrices from the amounts accumulated over time in POCIS, data are needed on the sampling rates $(\mathrm{L} / \mathrm{d})$ of each target compound into the passive sampler. Laboratory-based calibration of POCIS was conducted in order to assess the sampling rates for the opioids, cocaine and amphetamines included in this study.

In order to achieve the objectives of this study, 24-h composite samples were collected and POCIS were deployed in untreated and treated wastewater at the municipal wastewater treatment plants (WWTPs) serving two very different Canadian cities. The city served by WWTP-s is a small urban center in a primarily rural area of the province of Ontario with a population of 75,000 , with a demographic that is older and less diverse than larger communities in the province. The city served by WWTP-L is a large urban center of 1.6 million in the province of Quebec with a relatively young and ethnically diverse population. Data collected for prescription opioids and illicit drugs in municipal wastewater using 24-h composite sampling and passive sampling (i.e. POCIS) regimes were used to estimate community drug consumption patterns.

\section{Experimental}

\subsection{Chemicals}

Analytical standards of all target chemicals and stable isotope internal standards listed in Table 1 (purity $>99 \%$, isotopic purity $>94 \%$ ) were prepared individually in methanol and stored in amber vials at $-18{ }^{\circ} \mathrm{C}$ for less than 4 months. The deuterated stable isotope surrogates of each model compound were used for quantitation. In cases where no labeled surrogates were available, the surrogate with the closest chromatographic retention time to the model compound was selected for quantitation (Table 1). Suppliers of the standards are provided in the footnotes in Table 1. High performance liquid chromatography (HPLC grade or equivalent) methanol, acetone, acetonitrile, ACS reagent grade dichloromethane (DCM), hydrochloride acid (37\%), sulphuric acid (96\%), formic acid (88\%), and ammonium hydroxide (30\%) were purchased from Fisher Scientific (Ottawa, ON, Canada).

\subsection{POCIS calibration}

A laboratory-based calibration experiment was performed to determine the POCIS sampling rate of each target compound. Procedures for this experiment were based on work previously reported by Macleod et al. (2007), with some modifications reported by Li et al. (2010a). Briefly, static experiments were conducted in triplicate in 4-L amber glass bottles situated in a temperature-controlled environmental chamber set to $20^{\circ} \mathrm{C}$ for a period of eight days. The negative control contained deionized (MilliQ) water but was not spiked with the test compounds. The positive control contained deionized water and the target compound
Table 1

Target compounds analyzed in wastewater, their molecular weight and internal standard used for quantitation by LC-MS/MS. Chemical suppliers are listed in the footnotes.

\begin{tabular}{|c|c|c|}
\hline Target compound & MW & Internal standard (surrogate) \\
\hline \multicolumn{3}{|l|}{ Cocaine and metabolite } \\
\hline Cocaine $^{4}$ & 303.4 & Cocaine- $\mathrm{d}_{3}^{4}$ \\
\hline Benzoylecgonine $^{4}$ & 289.3 & Benzoylecgonine- $\mathrm{d}_{8}{ }^{4}$ \\
\hline \multicolumn{3}{|c|}{ Amphetamine-type stimulants } \\
\hline Amphetamine 4 & 135.2 & Amphetamine- $\mathrm{d}_{8}{ }^{4}$ \\
\hline $\mathrm{MDA}^{4}$ & 179.2 & MDA- $\mathrm{d}_{5}^{4}$ \\
\hline Methamphetamine $^{4}$ & 149.2 & Methamphetamine- $\mathrm{d}_{9}{ }^{4}$ \\
\hline $\mathrm{MDMA}^{4}$ & 193.2 & MDMA-d $_{5}^{4}$ \\
\hline Ephedrine $^{1}$ & 165.2 & Ephedrine- $\mathrm{d}_{3}{ }^{1}$ \\
\hline \multicolumn{3}{|l|}{ Opioid drugs } \\
\hline Codeine $^{1}$ & 299.4 & Codeine- $\mathrm{d}_{6}{ }^{1}$ \\
\hline Acetylcodeine $^{1}$ & 341.4 & Heroin- $d_{9}{ }^{1}$ \\
\hline Dihydrocodeine $^{1}$ & 301.4 & Dihydrocodeine- $\mathrm{d}_{6}{ }^{1}$ \\
\hline Morphine $^{1}$ & 285.3 & Morphine- $\mathrm{d}_{6}{ }^{1}$ \\
\hline 6-Acetylmorphine ${ }^{1}$ & 327.4 & 6 -Acetylmorphine- $\mathrm{d}_{6}{ }^{1}$ \\
\hline Methadone ${ }^{1}$ & 309.4 & Methadone- $d_{9}^{1}$ \\
\hline Heroin $^{1}$ & 369.4 & Heroin- $\mathrm{d}_{9}{ }^{1}$ \\
\hline Tramadol $^{2}$ & 263.4 & Tramadol- $\mathrm{d}_{6}{ }^{3}$ \\
\hline Ketamine $^{1}$ & 237.7 & Oxycodone- $\mathrm{d}_{6}{ }^{1}$ \\
\hline Oxycodone ${ }^{1}$ & 315.4 & Oxycodone- $\mathrm{d}_{6}{ }^{1}$ \\
\hline EDDP perchlorate $^{1}$ & 277.1 & EDDP perchlorate- $\mathrm{d}_{3}{ }^{1}$ \\
\hline
\end{tabular}

1 Cambridge.

2 Sigma.

3 CDN.

${ }^{4}$ Cerilliant.

standards, but no POCIS. The POCIS were soaked in deionized water overnight before the beginning of the experiment in order to decrease the possibility of a greater flux across the membrane when it first gets wet. A magnetic stirrer was used to gently mix the water, set to a stirring speed of approximately 800 to $900 \mathrm{rpm}$. The negative control was not stirred. Every hour over an 8-day period, $20 \mathrm{~mL}$ aliquots of water were removed from each vessel to monitor the decrease in water concentration over time and extracted within $24 \mathrm{~h}$ using the solid-phase extraction (SPE) method described below.

The sampling rate of the target chemicals by POCIS following firstorder kinetics was calculated using a linear regression describing the loss of a compound from water as a result of POCIS uptake over the 8d duration of the calibration study. The uptake rate constants were calculated for individual triplicate experiments from plots of the lntransformed concentrations ( $\mathrm{y}$-axis) over time ( $\mathrm{x}$-axis). The average rate constant, $\mathrm{k}$ was calculated as the average $( \pm S D)$ of the experiments conducted in triplicate. At the end of the calibration experiment, the POCIS disks were removed from the test chambers and extracted as described below to compare accumulation of the test compounds into the POCIS sorbent relative to the uptake rates determined from the loss from water over time. The analysis of these results confirmed that uptake of the target analytes by the membrane had a negligible effect on the sampling rates determined.

\subsection{Sampling in WWTPS}

Wastewater samples were collected and POCIS samplers were deployed over a two-week period at the WWTPs serving two cities in Canada. The sample collection period at WWTP-s (population 75,000, activated sludge treatment, $45,000 \mathrm{~m}^{3} / \mathrm{d}$ ) was over November 18 to December 2, 2010, and the sample collection period at WWTP-L (population $1.5 \mathrm{M}$, advanced primary TREATMENT, 2,000,000 $\mathrm{m}^{3} / \mathrm{d}$ ) was over January 18 to 31, 2011.

Samples ( $1 \mathrm{~L}$ ) of untreated and treated wastewater were collected as 24-h composites (equal volume, every hour). At WWTP-s, wastewater samples were collected only at the dates of deployment (Nov. 18) and 
retrieval (Dec. 2). At WWTP-L, wastewater samples were collected at deployment (Jan. 18) and thereafter every 2 days until the end of the deployment period (Jan. 31), for a total of 8 samples. The POCIS samplers were kept in air-tight canisters prior to deployment and were transferred to stainless-steel deployment cages at the WWTPs. Two cages were deployed in untreated and treated wastewater at each WWTP, with each cage containing six POCIS devices. POCIS field blanks were exposed to air at deployment and retrieval.

The 24-h composite samples of untreated and treated wastewater were extracted within 24-h of collection using the solid phase extraction procedures described below. POCIS devices were retrieved at the end of the deployment period and individually wrapped in aluminum foil on site. Each disk was stored in a plastic re-sealable bag and stored on ice during transportation back to the laboratory. To ensure that analytes were retained on the POCIS, samplers were stored in a freezer at $-20{ }^{\circ} \mathrm{C}$ (Carlson et al., 2013), and extraction was performed was within one month of retrieval.

\subsection{Extraction of aqueous samples}

Samples of water collected from the POCIS calibration experiment (i.e. $20 \mathrm{~mL}$ ) or aliquots of 24 -h composite samples of wastewater collected from the WWTPs (i.e. $100 \mathrm{~mL}$ for untreated wastewater, $200 \mathrm{~mL}$ for treated wastewater) were extracted using SPE. Prior to extraction, the WWTP field samples were filtered through $1-\mu \mathrm{m}$ glass fiber filters to remove particulates. Oasis MCX cartridges were preconditioned sequentially with $6 \mathrm{~mL}$ of acetone, methanol and deionized (milli-Q) water at $\mathrm{pH} 2.5$. All water samples were adjusted to a $\mathrm{pH}$ of 2.5 using $3.5 \mathrm{M} \mathrm{H}_{2} \mathrm{SO}_{4}$ and then spiked with $100 \mu \mathrm{L}$ of an internal standard mixture containing $500 \mathrm{ng} / \mathrm{mL}$ of each surrogate compound (Table 1). The samples were loaded onto the MCX cartridges at a rate of $1 \mathrm{~mL} / \mathrm{min}$. After aspiration to dryness, the SPE cartridges were eluted into conical centrifuge tubes with three sequential $3 \mathrm{~mL}$ rinsings of $5 \%$ ammonium hydroxide in methanol. The eluent was then evaporated to near-dryness using a rotational vacuum concentrator and reconstituted to a final volume of $0.4 \mathrm{~mL}$ in methanol for analysis. Procedural blanks were extracted concurrently with water samples. The coefficients of variation were $3-5 \%$ and the recoveries were $83-101 \%$ for the target analytes.

\subsection{Extraction of POCIS}

POCIS samplers collected from the municipal WWTP and from the calibration experiment were extracted as described previously by $\mathrm{Li}$ et al. (2010a). Briefly, the POCIS samplers were removed from the freezer and rinsed gently under water. Each POCIS sampler was disassembled and the sorbent was transferred to a glass column fitted with a glass wool plug and approximately $5 \mathrm{~cm}$ of granular anhydrous sodium sulfate. The sorbent in the column was spiked with $100 \mu \mathrm{L}$ of the internal standard mixture containing $500 \mathrm{ng} / \mathrm{mL}$ of each surrogate compound (Table 1). The column was then eluted with $100 \mathrm{~mL}$ of methanol into a round bottom flask. The eluate was evaporated to approximately $1 \mathrm{~mL}$ via rotary evaporation and then transferred to a conical centrifuge tube using three $1 \mathrm{~mL}$ methanol rinses. Samples were evaporated to near dryness and brought to a final volume of $1.0 \mathrm{~mL}$ in 1:1 methanol/ water.

\subsection{Analysis}

All extracts were analyzed by liquid chromatography and tandem mass spectrometry with electrospray ionization (LC-ESI-MS/MS) using a Shimadzu HPLC coupled to an Applied Biosystems API 3000 tandem mass spectrometer (MDS Sciex, Concord, ON, Canada). The mass spectrometer was operated in positive or negative ion mode using multiple-reaction monitoring (MRM) for the transition ions with the conditions presented in the Supplemental material, Table S1.
Two LC-MS/MS methods were used for the analysis of the target compounds from the illicit drug and opioid drug classes using the chromatographic and ionization conditions listed in the Supplemental material, Table S2. Analytes were separated chromatographically on a Genesis C18 column $(150 \times 2.1 \mathrm{~mm}$ i.d., $4 \mu \mathrm{m}$ particle size; Chromatographic Specialties, Brockville, ON, Canada) coupled with a guard column with the same packing material $(4 \mathrm{~mm} \times 2.0 \mathrm{~mm}$; Phenomenex, Torrance, CA, USA). Stable isotope surrogates (Table 1) were used to compensate for matrix-induced signal suppression and recoveries. The LODs and LOQs for the target analytes in wastewater are listed in Table S1. Procedural blanks and quality controls were run for each set of 10 samples.

\subsection{Estimates of community drug use}

A previously described method (Zuccato et al., 2008) was used to estimate community use of the target drugs. The amount of drug discharged $(\mathrm{g} / \mathrm{d})$ was calculated using the mean $(\mathrm{n}=3)$ concentration of the target drug in the 24-h composite samples of untreated wastewater and the daily flow rate in the WWTPs. This value was then divided by the number of people served by the WWTP to estimate the grams of drug excreted in wastewater per person per day. This value was then normalized to a value of grams per day per 1000 people. To obtain a consumption value for each drug within the community, the fractions of the target drugs excreted in urine were used (Table 2). In order to determine community drug use (i.e. doses/d/1000 people), the consumption value was divided by an amount considered typical for a single dose of the target drug. The typical doses of illicit drugs or the recommended therapeutic doses of prescription drugs are presented in Table 2 .

\section{Results and discussion}

\subsection{Calibration of POCIS}

POCIS sampling rates (Rs) were determined for 14 of the target compounds and are summarized in Table 3. These include the first sampling rates reported for 7 compounds (MDA, ephedrine, dihydrocodeine, methadone, tramadol, oxycodone and EDDP). For codeine, a comparable value of $0.329 \pm 0.133 \mathrm{~L} / \mathrm{d}$ was reported by MacLeod et al. (2007). For the other compounds, Harman et al. (2011) reported similar sampling rates for cocaine (average $0.150 \mathrm{~L} / \mathrm{d}$ ) but lower sampling rates for the other compounds. This difference might be explained by the in situ calibration approach used by Harman et al. (2011). The sampling rates for acetylmorphine, acetylcodeine and heroin could not be determined because of their low measured uptake and poor linearity $\left(\mathrm{R}^{2}<0.90\right)$. A possible explanation for the low compatibility of the sorbent and POCIS configuration used with heroin, acetylcodeine and

Table 2

Parameters used to estimate community drug use.

\begin{tabular}{llllc}
\hline Drugs & $\begin{array}{l}\text { Analytical target } \\
\text { (AT) }\end{array}$ & $\begin{array}{l}\text { Percentage of } \\
\text { drug excreted } \\
\text { as AT }\end{array}$ & $\begin{array}{l}\text { Molar mass } \\
\text { ratio } \\
\text { (drug AT) }\end{array}$ & $\begin{array}{l}\text { Typical } \\
\text { dose } \\
\text { (mg) }\end{array}$ \\
\hline $\begin{array}{llll}\text { Amphetamine } \\
\text { Methamphetamine }\end{array}$ & Amphetamine & $30 \%^{1}$ & 1.0 & 30 \\
Cocaine & Methamphetamine & $43 \%^{1}$ & 1.0 & 30 \\
MDMA & MDMA & $65 \%^{1}$ & 1.05 & 100 \\
Ephedrine & Ephedrine & $75 \%^{2}$ & 1.0 & 100 \\
Heroin & Morphine & $42 \%^{1}$ & 1.0 & 30 \\
Oxycodone & Oxycodone & $14 \%^{3}$ & 1.29 & 25 \\
Ketamine & Ketamine & $30 \%^{3}$ & 31.0 & 25 \\
Tramadol & Tramadol & $30 \%^{4}$ & 1.0 & 75 \\
\hline
\end{tabular}

1 Zuccato et al., 2008.

2 Postigo et al., 2011.

3 http://www.drugs.com/.

${ }^{4}$ http://opiods.com/. 
Table 3

Mean $( \pm \mathrm{SD})$ sampling rates, Rs $(\mathrm{L} / \mathrm{d})$ of target analytes $(n=6)$.

$\mathrm{NA}=$ sampling rates could not be determined from the experimental data.

\begin{tabular}{ll}
\hline Analyte & Rs (L/d) \\
\hline $\begin{array}{l}\text { Cocaine and its metabolite } \\
\text { Cocaine }\end{array}$ & $0.130 \pm 0.036$ \\
Benzoylecgonine & $0.134 \pm 0.011$ \\
Amphetamine-type stimulants & \\
Amphetamine & $0.201 \pm 0.038$ \\
MDA & $0.288 \pm 0.021$ \\
Methamphetamine & $0.231 \pm 0.025$ \\
MDMA & $0.222 \pm 0.013$ \\
Ephedrine & $0.123 \pm 0.039$ \\
Opioid drugs & \\
Codeine & \\
Acetylcodeine & $0.394 \pm 0.049$ \\
Dihydrocodeine & $\mathrm{NA}$ \\
Morphine & $0.110 \pm 0.041$ \\
Acetylmorphine & $0.261 \pm 0.036$ \\
Methadone & $\mathrm{NA}$ \\
Heroin & $0.408 \pm 0.147$ \\
Tramadol & $\mathrm{NA}$ \\
Oxycodone & $0.241 \pm 0.062$ \\
EDDP & $0.152 \pm 0.039$ \\
\hline
\end{tabular}

acetylmorphine could be due to their acetylated structures, giving a high polarity and an affinity for water over the sorbent. Previous work has also indicated that extremely polar compounds don't sorb to POCIS sequestration media (Alvarez et al., 2007).

Mass balance calculations were conducted to see if the amount of the target analytes adsorbed to the POCIS sorbent accounted for the amount of these compounds lost from water over the course of the static experiment. With the exception of heroin, acetylcodeine and acetylmorphine, the mass of the test compounds adsorbed onto the POCIS sorbent accounted for between 82 and 95\% of the amount removed from water over the 8-d calibration experiment. These data indicate that the target analytes were efficiently sequestered into the sorbent and were not accumulating in the POCIS membrane. In a recent review of the POCIS monitoring technique, Morin et al. (2012) concluded that the static uptake method used in the present study was a valid method for calibrating POCIS.

\subsection{Levels of prescription opioids and illicit drugs in wastewater}

Tables 4 and 5 summarize the frequency of detection, average and maximum concentrations ( $\mathrm{ng} / \mathrm{L}$ ) of the target analytes measured in the untreated and treated water using 24-h composite samples and POCIS, respectively, for each wastewater treatment plant. Note that the data for concentrations from POCIS sampling were estimated from the amounts (ng) accumulated in the passive samplers over the 14-d deployment periods and the sampling rates (L/d). All the 19 target drugs were detected in the 24-h composite samples of untreated and treated wastewater, with the exception of heroin, acetylcodeine and acetylmorphine. These results may have been due to the relatively high LODs for these compounds (Table S1) and low concentrations in the water samples. In addition, heroin is rapidly biotransformed and excreted as morphine and acetylmorphine (Trescot et al., 2008). MDA was not detected in the 24-h composite samples but was detected in the POCIS, while the opposite trend was observed for methamphetamine and tramadol. The highest concentrations observed in the untreated wastewater from both WWTPs corresponded to cocaine and its metabolite, benzoylecgonine. The highest levels in the treated wastewater corresponded to codeine for WWTP-s and to benzoylecgonine for WWTP-L. In general, the levels observed in the present study are consistent with those reported in Europe by other researchers (Boleda et al., 2007, 2009; Postigo et al., 2010; Terzic et al., 2010; van Nuijs et al., 2011a) and in our previous study on illicit drugs in Canadian cities (Metcalfe et al., 2010).

There are some interesting trends in comparing the data for untreated wastewater from the two cities (Tables 4 and 5). The levels of cocaine, benzoylecgonine and methamphetamine were higher in wastewater from the larger urban center (i.e. WWTP-L). Maximum and mean concentrations of MDMA (ecstasy) appeared to be similar in wastewater from the two cities. In untreated wastewater from the smaller urban center (i.e. WWTP-s), the mean and maximum levels of oxycodone and codeine were significantly higher than in untreated wastewater from the larger urban center (i.e. WWTP-L), for both the 24-h composite and passive sampling methods. The elevated levels of these prescription opioids may reflect the older population base served by WWTP-s, or in the case of oxycodone, may reflect abuse of this drug. Ketamine was detected only in untreated wastewater from WWTP-L using both sampling methods. Both mean and maximum concentrations of ephedrine were slightly higher in the 24-h composite samples of wastewater from WWTP-L. The analytical results for EDDP, tramadol

Table 4

Frequency of detection (\%) and average and maximum concentration (ng/L) of drugs measured in the untreated and treated wastewater based on the 24-h composite samples.

\begin{tabular}{|c|c|c|c|c|c|c|c|c|c|c|c|c|c|c|c|c|}
\hline \multirow[t]{4}{*}{ Compounds } & \multicolumn{8}{|c|}{ Untreated wastewater } & \multicolumn{8}{|c|}{ Treated wastewater } \\
\hline & \multicolumn{2}{|c|}{$\begin{array}{l}\text { Frequency of } \\
\text { detection (\%) }\end{array}$} & \multicolumn{4}{|c|}{ Average concentration (ng/L) } & \multicolumn{2}{|c|}{$\begin{array}{l}\text { Maximum } \\
\text { concentration (ng/L) }\end{array}$} & \multicolumn{2}{|c|}{$\begin{array}{l}\text { Frequency of } \\
\text { detection (\%) }\end{array}$} & \multicolumn{4}{|c|}{ Average concentration (ng/L) } & \multicolumn{2}{|c|}{$\begin{array}{l}\text { Maximum } \\
\text { concentration (ng/L) }\end{array}$} \\
\hline & \multirow{2}{*}{$\frac{\text { WWTP-S }}{n=6}$} & \multirow{2}{*}{$\frac{\text { WWTP-L }}{\mathrm{n}=4}$} & \multicolumn{2}{|c|}{ WWTP-S } & \multicolumn{2}{|c|}{ WWTP-L } & \multirow[t]{2}{*}{ WWTP-S } & \multirow[t]{2}{*}{ WWTP-L } & \multirow{2}{*}{$\frac{\text { WWTP-S }}{\mathrm{n}=6}$} & \multirow{2}{*}{$\frac{\text { WWTP-L }}{\mathrm{n}=4}$} & \multicolumn{2}{|c|}{ WWTP-S } & \multicolumn{2}{|c|}{ WWTP-L } & \multirow[t]{2}{*}{ WWTP-S } & \multirow[t]{2}{*}{ WWTP-L } \\
\hline & & & AVG & $\pm \mathrm{SD}$ & AVG & $\pm \mathrm{SD}$ & & & & & AVG & $\pm \mathrm{SD}$ & AVG & $\pm S D$ & & \\
\hline Amphetamine & $100 \%$ & $100 \%$ & 43 & \pm 5 & 23 & \pm 6 & 60 & 37 & $50 \%$ & $100 \%$ & 6 & \pm 2 & 21 & \pm 1 & 13 & 25 \\
\hline MDA & $0 \%$ & $0 \%$ & nd & & nd & & nd & nd & $0 \%$ & $0 \%$ & nd & & nd & & nd & nd \\
\hline Methamphetamine & $0 \%$ & $100 \%$ & nd & & 18 & \pm 5 & nd & 28 & $0 \%$ & $100 \%$ & nd & & 29 & \pm 7 & nd & 42 \\
\hline MDMA & $100 \%$ & $100 \%$ & 131 & \pm 2 & 69 & \pm 6 & 138 & 108 & $100 \%$ & $100 \%$ & 67 & \pm 5 & 85 & \pm 6 & 82 & 128 \\
\hline Ephedrine & $100 \%$ & $100 \%$ & 100 & \pm 11 & 363 & \pm 40 & 202 & 381 & $100 \%$ & $100 \%$ & 231 & \pm 16 & 510 & \pm 50 & 421 & 616 \\
\hline Benzoylecgonine & $100 \%$ & $100 \%$ & 241 & \pm 29 & 1247 & \pm 128 & 273 & 1592 & $100 \%$ & $100 \%$ & 471 & \pm 73 & 1182 & \pm 111 & 656 & 1446 \\
\hline Cocaine & $100 \%$ & $100 \%$ & 115 & \pm 8 & 348 & \pm 56 & 137 & 412 & $100 \%$ & $100 \%$ & 80 & \pm 8 & 245 & \pm 47 & 118 & 457 \\
\hline Morphine & $100 \%$ & $100 \%$ & 30 & \pm 5 & 17 & \pm 8 & 43 & 28 & $100 \%$ & $100 \%$ & 48 & \pm 5 & 23 & \pm 3 & 65 & 29 \\
\hline Dihydrocodeine & $67 \%$ & $0 \%$ & 0.2 & \pm 0.2 & nd & & 1 & nd & $100 \%$ & $0 \%$ & 0.4 & \pm 0.4 & nd & & 1 & nd \\
\hline Codeine & $100 \%$ & $100 \%$ & 513 & \pm 25 & 66 & \pm 15 & 807 & 88 & $100 \%$ & $100 \%$ & 795 & \pm 32 & 50 & \pm 6 & 1230 & 69 \\
\hline Oxycodone & $100 \%$ & $0 \%$ & 31 & \pm 0.7 & nd & & 43 & nd & $100 \%$ & $0 \%$ & 45 & \pm 0.4 & nd & & 61 & nd \\
\hline Ketamine & $0 \%$ & $100 \%$ & nd & & 40 & \pm 4 & nd & 52 & $0 \%$ & $100 \%$ & nd & & 41 & \pm 9 & nd & 57 \\
\hline Tramadol & $100 \%$ & $100 \%$ & 29 & \pm 3 & 18 & \pm 2 & 44 & 21 & $100 \%$ & $100 \%$ & 59 & \pm 5 & 37 & \pm 4 & 88 & 43 \\
\hline EDDP & $100 \%$ & $100 \%$ & 75 & \pm 1 & 115 & \pm 37 & 103 & 227 & $100 \%$ & $100 \%$ & 106 & \pm 4 & 27 & \pm 3 & 148 & 112 \\
\hline Methadone & $100 \%$ & $96 \%$ & 28 & \pm 0.4 & 1 & \pm 0.2 & 38 & 2 & $100 \%$ & $100 \%$ & 43 & \pm 0.8 & 2 & \pm 0.5 & 62 & 3 \\
\hline
\end{tabular}


Table 5

Frequency of detection (\%) and average and maximum concentration (ng/L) of drugs measured in the untreated and treated wastewater based on the POCIS analysis.

\begin{tabular}{|c|c|c|c|c|c|c|c|c|c|c|c|c|c|c|c|c|}
\hline \multirow[t]{4}{*}{ Compounds } & \multicolumn{8}{|c|}{ Untreated wastewater } & \multicolumn{8}{|c|}{ Treated wastewater } \\
\hline & \multicolumn{2}{|c|}{$\begin{array}{l}\text { Frequency of } \\
\text { detection (\%) }\end{array}$} & \multicolumn{4}{|c|}{$\begin{array}{l}\text { Average concentration } \\
\text { (ng/L) }\end{array}$} & \multicolumn{2}{|c|}{$\begin{array}{l}\text { Maximum } \\
\text { concentration (ng/L) }\end{array}$} & \multicolumn{2}{|c|}{$\begin{array}{l}\text { Frequency of } \\
\text { detection (\%) }\end{array}$} & \multicolumn{4}{|c|}{ Average concentration (ng/L) } & \multicolumn{2}{|c|}{$\begin{array}{l}\text { Maximum } \\
\text { concentration (ng/L) }\end{array}$} \\
\hline & \multirow{2}{*}{$\frac{\text { WWTP-S }}{n=6}$} & \multirow{2}{*}{$\frac{\text { WWTP-L }}{n=4}$} & \multicolumn{2}{|c|}{ WWTP-S } & \multicolumn{2}{|c|}{ WWTP-L } & \multirow[t]{2}{*}{ WWTP-S } & \multirow[t]{2}{*}{ WWTP-L } & \multirow{2}{*}{$\frac{\text { WWTP-S }}{n=6}$} & \multirow{2}{*}{$\frac{\text { WWTP-L }}{n=4}$} & \multicolumn{2}{|c|}{ WWTP-S } & \multicolumn{2}{|c|}{ WWTP-L } & \multirow[t]{2}{*}{ WWTP-S } & \multirow[t]{2}{*}{ WWTP-L } \\
\hline & & & AVG & $\pm \mathrm{SD}$ & AVG & $\pm \mathrm{SD}$ & & & & & AVG & $\pm \mathrm{SD}$ & AVG & $\pm \mathrm{SD}$ & & \\
\hline Amphetamine & $100 \%$ & $100 \%$ & 7 & \pm 2 & 56 & \pm 11 & 10 & 70 & $50 \%$ & $100 \%$ & 1 & \pm 0.1 & 20 & \pm 3 & 1 & 22 \\
\hline MDA & $0 \%$ & $0 \%$ & nd & & nd & & nd & nd & $0 \%$ & $0 \%$ & nd & & nd & & nd & nd \\
\hline Methamphetamine & $100 \%$ & $100 \%$ & 2 & \pm 0.4 & 13 & \pm 4 & 3 & 16 & $100 \%$ & $100 \%$ & 3 & \pm 1.2 & 18 & \pm 2 & 5 & 20 \\
\hline MDMA & $100 \%$ & $100 \%$ & 65 & \pm 25 & 88 & \pm 2 & 122 & 89 & $100 \%$ & $100 \%$ & 67 & \pm 11 & 67 & \pm 10 & 82 & 75 \\
\hline Ephedrine & $100 \%$ & $100 \%$ & 112 & \pm 22 & 133 & \pm 13 & 151 & 154 & $100 \%$ & $100 \%$ & 14 & \pm 4 & 152 & \pm 8 & 19 & 160 \\
\hline Benzoylecgonine & $100 \%$ & $100 \%$ & 306 & \pm 66 & 60 & \pm 5 & 388 & 65 & $100 \%$ & $100 \%$ & 101 & \pm 15 & 76 & \pm 9 & 116 & 76 \\
\hline Cocaine & $100 \%$ & $100 \%$ & 122 & \pm 37 & 38 & \pm 4 & 177 & 24 & $100 \%$ & $100 \%$ & 204 & \pm 65 & 81 & \pm 9 & 324 & 53 \\
\hline Morphine & $100 \%$ & $100 \%$ & 18 & \pm 5 & 2 & \pm 1 & 24 & 4 & $100 \%$ & $100 \%$ & 13 & \pm 3 & 4 & \pm 0.5 & 18 & 4 \\
\hline Dihydrocodeine & $100 \%$ & $100 \%$ & na & & na & & na & na & $100 \%$ & $100 \%$ & na & & na & & na & na \\
\hline Codeine & $100 \%$ & $100 \%$ & 177 & \pm 34 & 22 & \pm 6 & 234 & 31 & $100 \%$ & $100 \%$ & 893 & \pm 208 & 15 & \pm 3 & 1139 & 18 \\
\hline Oxycodone & $100 \%$ & $100 \%$ & 66 & \pm 21 & 1 & \pm 0.1 & 93 & 10 & $100 \%$ & $100 \%$ & 220 & \pm 15 & 9 & \pm 0.5 & 235 & 10 \\
\hline Ketamine & $0 \%$ & $100 \%$ & na & & na & & na & na & $100 \%$ & $75 \%$ & na & & na & & na & na \\
\hline Tramadol & $0 \%$ & $0 \%$ & nd & & nd & & nd & nd & $100 \%$ & $0 \%$ & 164 & \pm 13 & nd & & 179 & nd \\
\hline EDDP & $0 \%$ & $100 \%$ & nd & & 15 & \pm 1 & nd & 15 & $100 \%$ & $100 \%$ & 193 & \pm 57 & 11 & \pm 0.4 & 254 & 12 \\
\hline Methadone & $0 \%$ & $100 \%$ & nd & & 2 & \pm 0.2 & nd & 3 & $100 \%$ & $100 \%$ & 128 & \pm 37 & 9 & \pm 0.3 & 171 & 9 \\
\hline
\end{tabular}

and methadone are inconsistent for samples collected as 24-h composites or with POCIS. For the POCIS samples, EDDP and methadone were only detected in WWTP-L, and tramadol was not detected at either plant. For the 24-h composite samples, EDDP, tramadol and methadone were detected at relatively low concentrations in untreated wastewater from both plants.

\subsection{Comparison of passive samplers to composite sampling}

In order to evaluate the use of POCIS for quantification of prescription opioids and drugs of abuse in wastewater, the concentrations estimated from the POCIS were compared to values obtained using 24-h composite samples collected upon deployment and retrieval of the passive samplers. We acknowledge that data derived from a limited number of composite samples may not be representative of the exposures for POCIS deployed over 2 weeks. These comparisons give an indication whether the POCIS data are within the range of concentrations that have been directly measured in wastewater, but the data should be interpreted with caution. As shown in Figs. 1 and 2 for both untreated and treated water, respectively at both WWTPs, the concentrations estimated from POCIS were similar to the values obtained using composite sampling at the time of deployment and retrieval. However, for some of the opioid drugs, and especially ketamine, tramadol, EDDP and methadone, the POCIS gave estimates of concentrations in the untreated
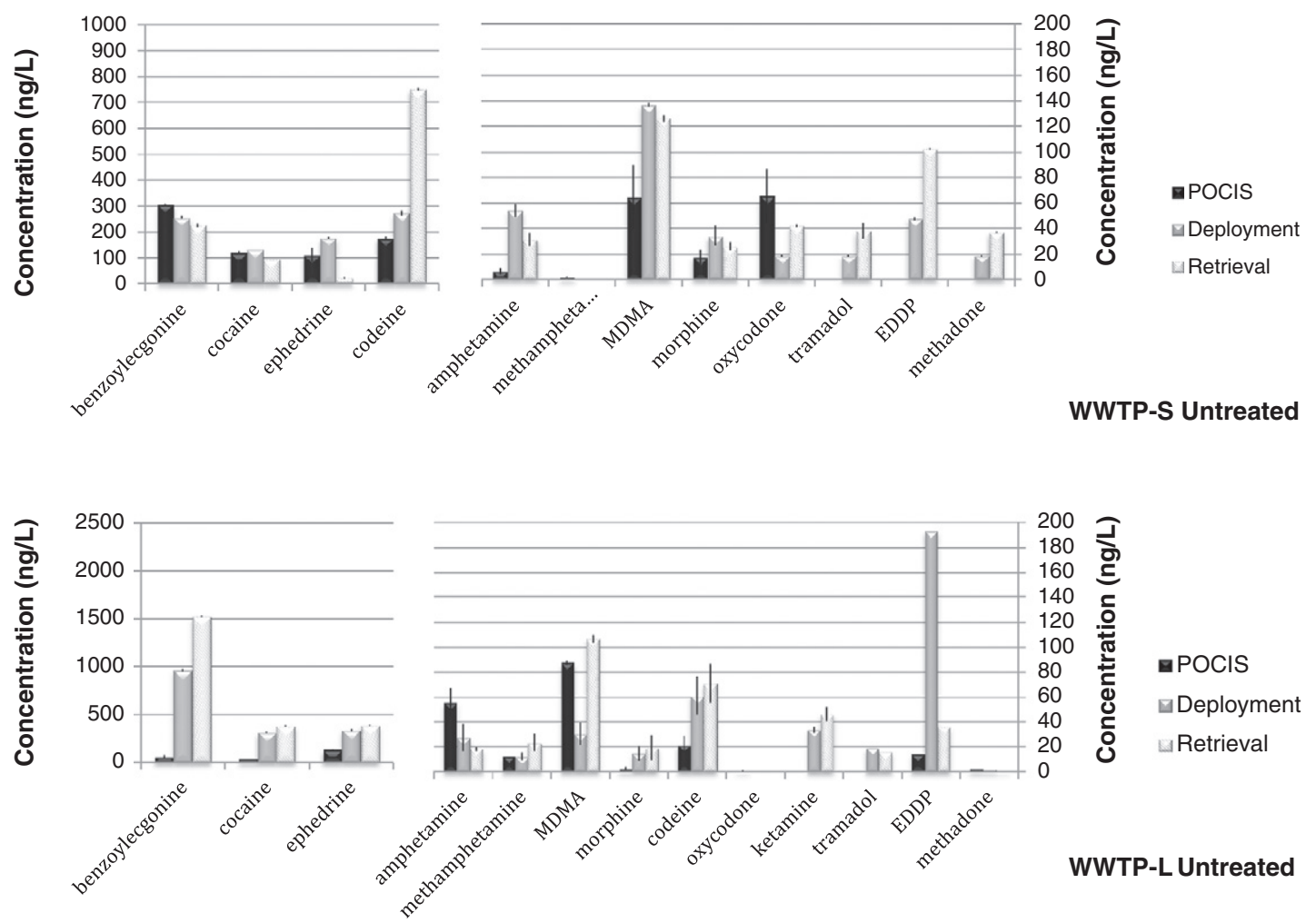

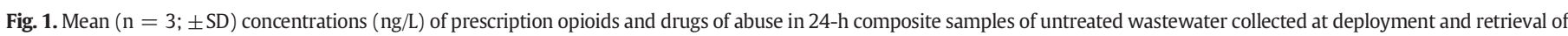

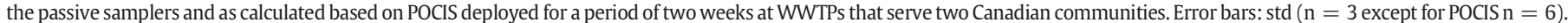



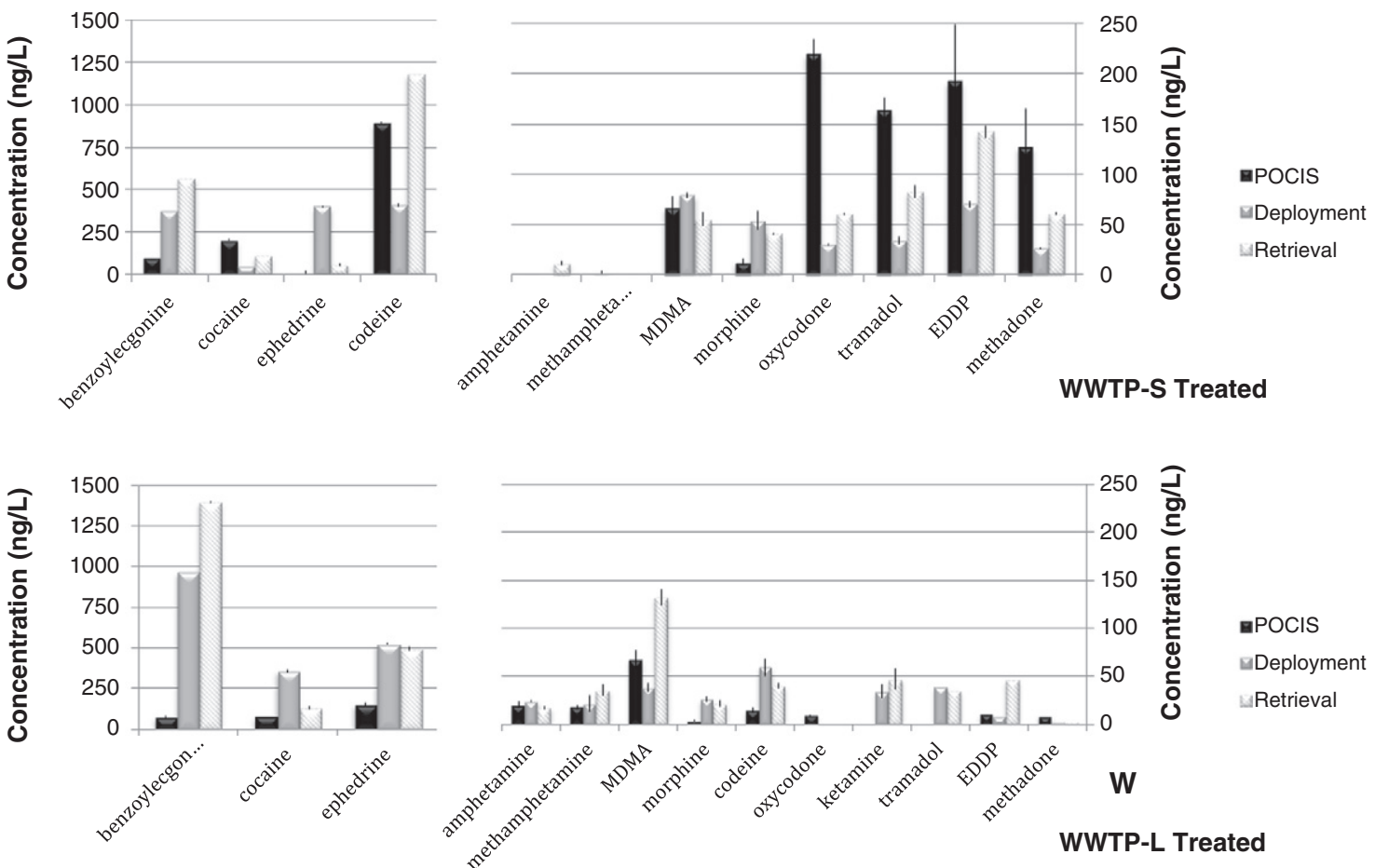

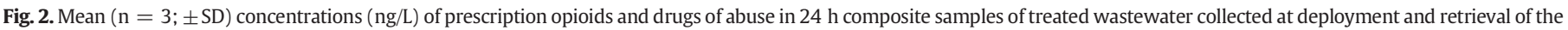

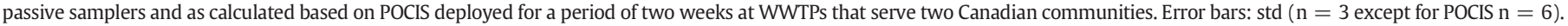

wastewater that were lower (or not detected), while it gave estimates in the treated wastewater that were higher than concentrations detected in composite samples. It is interesting that MDA was detected in the passive samplers, but not in the 24 -h composite samples. As mentioned previously, POCIS could not be used to estimate concentrations of acetylcodeine, acetylmorphine and heroin because sampling rates could not be calculated in our bench scale experiments. It appears that when the matrix is more complex (i.e. untreated wastewater), lower concentrations are calculated based on the POCIS results. In a few cases, such as for methamphetamine at WWTP-L, the drugs were not detected at all in the POCIS deployed in untreated wastewater. This might be explained by biofouling of the POCIS or clogging of the sampler cages by debris. In fact, larger discrepancies between the POCIS and composite samples were observed in WWTP-L where the treated wastewater matrix exiting the advanced primary treatment plant is more complex than the secondary effluent produced at WWTP-s. Curiously, Harman et al. (2009) observed an increase in the accumulation of alkylphenol compounds in POCIS after biofouling, which they explained as possible reductions in the interactions of the analytes with the fouled membrane. The role of biofouling in altering sampling rates for POCIS requires more study.

One source of variability using POCIS is the influence of temperature, flow and water quality parameters (e.g. pH, dissolved organic carbon) on the accumulation of target compounds. The estimates of concentrations in wastewater from POCIS data were based on sampling rates (Rs) determined in bench scale experiments conducted at $20^{\circ} \mathrm{C}$. The temperatures of the untreated wastewater at WWTP-S and WWTP-L were in this range over the sampling period, but temperatures of treated wastewater were in the range of $8-12{ }^{\circ} \mathrm{C}$. However, our previous studies have shown that sampling rates for POCIS do not vary widely with temperature, water flow, $\mathrm{pH}$ and dissolved organic content (Li et al., 2010a, 2010b, 2011). Overall, it appears that passive sampling using POCIS in complex matrices such as untreated wastewater would require different calibration approaches such as the in situ calibration suggested by Harman et al. (2011) in order to obtain data on concentrations that can be reliably used to estimate community drug consumption.
However, in some cases, POCIS may accumulate drugs to detectable levels when these compounds are not detectable in grab or composite samples of wastewater. Thus, passive sampling with POCIS may be a valuable tool for semi-quantitative monitoring of drugs of abuse in wastewater. Our previous studies have shown that POCIS provide reliable estimates of the concentrations of pharmaceuticals when deployed in surface waters (Li et al., 2010a).

\subsection{Removal of drugs by wastewater treatment}

Negative values for percent removals (data not shown) were observed for many of the target compounds, especially for the opioids. Similarly to Ternes (1998) who suggested that glucuronide conjugates formed during metabolism of drugs may be cleaved as these metabolites pass through WWTPs, thereby increasing the concentration of the parent compound in the treated wastewater, negative removals might be explained by the transformation of conjugated metabolites during biological treatment. These observations might also have been be due to mixing regime characteristics, as well as flow and concentration variability that may have led to negative elimination efficiencies, as demonstrated by Majewsky et al. (2011). In any event, Ort et al. (2010) pointed out the need for more sophisticated sampling strategies to accurately determine removals of drugs in wastewater treatment plants.

Concentration data generated from POCIS, integrating concentrations for the target drugs over a period of two weeks, did not give more consistent results for removals of most of the drugs relative to removals calculated from 24 -h composite samples. This may be due to the low efficiency of the POCIS for accumulating some of the opioid compounds, including tramadol, EDDP, methadone and ketamine. These discrepancies might be explained by fouling problems associated with sampling untreated wastewater leading to inappropriate sampling rates in that specific environment. Improved sampling strategies are required to better evaluate the removal of these drugs during wastewater treatment 

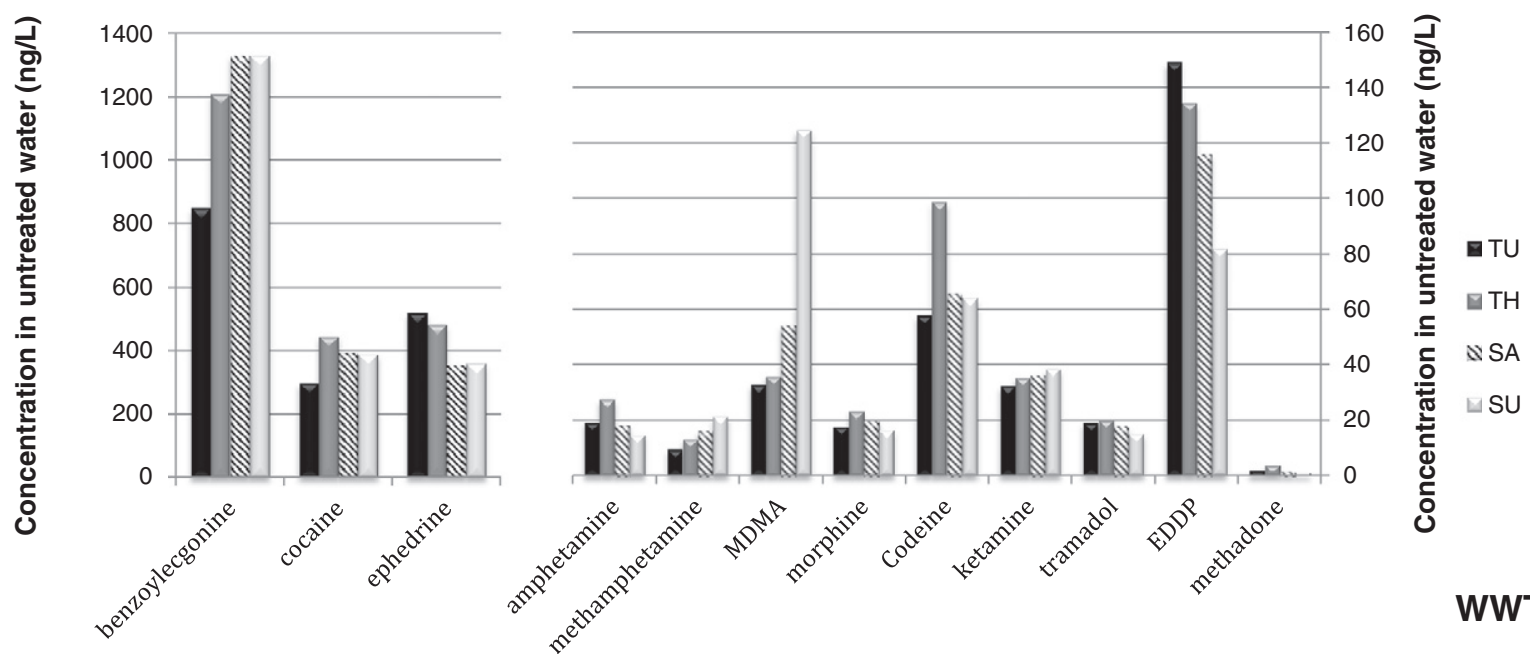

\section{WWTP-L}

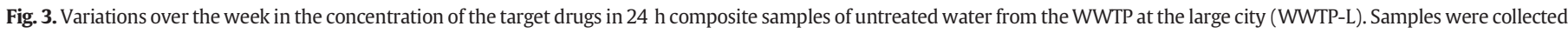
on Tuesday, Thursday, Saturday and Sunday on two successive weeks.

\subsection{Variations of drug concentrations over time}

As shown in Fig. 3, MDMA (i.e. Ecstasy) concentrations in 24-h composite samples of untreated wastewater from WWTP-L increased slightly on Saturday and markedly on Sunday relative to samples collected earlier in the week (i.e. Tuesday and Thursday). Ecstasy is known to be consumed by people who visit clubs, parties, music festivals and dance events (Yacoubian et al., 2003; McCaughan et al., 2005; Van Havere et al., 2011). These temporal variations strongly suggest that a sampling method accounting for episodic events is essential for adequate monitoring of these drugs in wastewater. Concentrations of other prescription opioids, amphetamines, cocaine and benzoylecgonine remained fairly consistent throughout the week, although there may have been a trend of increasing concentrations of methamphetamine as the week progressed. For reasons that are not clear, the concentrations of the prescription opioid, EDDP declined as the week progressed.

\subsection{Estimates of community drug use}

Community drug use was estimated for certain target compounds for which data are available on excretion rates of the parent compound or major metabolite and the estimated doses of illicit drugs or the recommended daily dosages of prescription drugs (Table 2 ). As presented in Fig. 4, the drug with the highest number of doses per 1000 people per day was cocaine, with a value of about 38 doses per day per 1000 in both communities. Cocaine use in the present study is within our previously published range of 8.1 to 56.7 (median 15.7) doses per day per 1000 people (Metcalfe et al., 2010). In our previous sampling of WWTP-s reported by Metcalfe et al. (2010), amphetamine was detected in untreated wastewater, while this illicit drug was not detected in the present study. Tramadol is being increasingly prescribed as a painkiller (Radbruch et al., 1996), so it is not surprising that there was moderate use of this drug in both communities (Fig. 4). Use of heroin (including therapeutic use of morphine) was low but consistent for the two communities. Since heroin use is estimated from levels of its metabolite, morphine, it is possible that illicit heroin use is overestimated as a result of the therapeutic use of morphine for palliative care and other medical uses. The community drug consumption values obtained in this study for cocaine, amphetamine, methamphetamine, MDMA (ecstasy) and heroin are similar to values reported for other countries (Castiglioni et al., 2006; Boleda et al., 2007; Zuccato et al., 2008; Kasprzyk-Hordern et al., 2009; Postigo et al., 2010; van Nuijs et al., 2011a, 2011b).

When comparing the data between WWTP-S and WWTP-L, the number of doses per day per 1000 people is fairly consistent for amphetamine, MDMA and heroin in the two cities (Fig. 4). There were

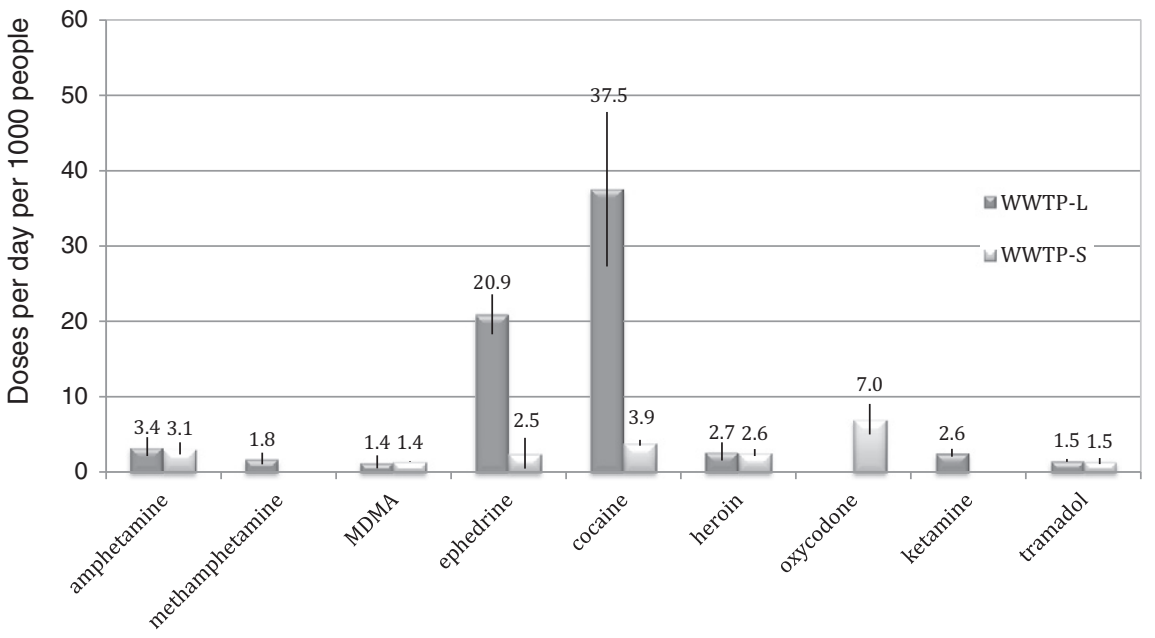

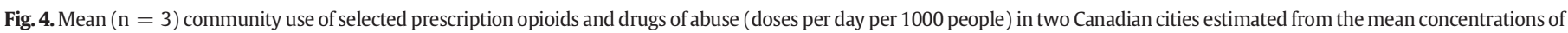
the target compounds in 24-h composite samples of untreated wastewater collected from the WWTPs that serve these communities. Error bars: std ( $\mathrm{n}=6$ ). 
differences between the two communities for drug consumption estimates for ephedrine, ketamine and oxycodone. This might be explained by the different population sizes and demographics of the two cities. WWTP-L serves a large, ethnically diverse metropolis with a median age of 38.8 (City-Data.com, 2012) and WWTP-s serves a smaller city in a rural region of Ontario with a median age of 42.8 (City-Data.com, 2012) and a high proportion $>60$ years old. The difference in average population age might explain the higher number of doses of codeine and oxycodone. Codeine is a commonly prescribed analgesic and oxycodone is prescribed for managing more severe pain, and sometimes is used for palliative care. However, elevated consumption of oxycodone in the city served by WWTP-s could also be due to abuse of this highly addictive drug. It has been documented that oxycodone is a major drug of abuse among high school students in the province of Ontario in Canada, as 3\% of students of 16 years of age reported using it for non-medical purposes (Paglia-Boak et al., 2011).

Ketamine was only detected in untreated wastewater from WWTP-L. This opioid is most commonly used as a veterinary tranquilizer but it is sometimes used for human surgical procedures. Because of its ability to produce a dissociative anesthetic state and induce hallucinations, ketamine has become an illicitly sold recreational drug in North America (Dotson et al., 1995). This is the first report of the presence of this drug in wastewater in a North American city and only the second report in the literature (van der Aa et al., 2010). Ephedrine, a substituted amphetamine, is a sympathomimetic agent that has been shown to increase energy in humans. Ephedrine is widely available in various forms, ranging from prescription and non-prescription medications to herbal products and energy drinks. Medical applications of ephedrine include treatment of asthma, sinusitis and acute hypertension. Ephedrine is also used in clandestine laboratories for production of methamphetamine (Lee et al., 2007), so some ephedrine in wastewater could be due to direct discharges from these clandestine sources. Postigo et al. (2010) found that out of 17 investigated compounds, ephedrine was the most prevalent in untreated wastewater from a treatment plant in Spain. In the city served by WWTP-L, ephedrine was second only to cocaine as having the highest community consumption at 21 doses per 1000 people. However, this consumption estimate probably underestimates community use of ephedrine because it is based upon the recommended therapeutic dose of $30 \mathrm{mg} / \mathrm{d}$ (Table 5).

The use of cocaine and methamphetamine in the city served by WWTP-S was lower than in the city served by WWTP-L (Fig. 4). The lower consumption in the smaller city may reflect problems with sustaining supplies of these illicit drugs. Law enforcement officers in the smaller city contend that supplies of cocaine and methamphetamines fluctuate widely in smaller centers because larger cities have greater demand. This may explain why smaller cities and rural areas are subject to abuse of prescription opioid drugs.

\section{Conclusions}

The survey of prescription opioids and illicit drugs in wastewater from WWTPs serving a large city and a small city in Canada shows that several opioids are present at detectable levels, but concentrations were all below the levels of cocaine and its major metabolite, benzoylecgonine. In terms of community drug consumption, cocaine had the highest level of consumption, but ephedrine was the next most prevalent compound in one of the WWTPs that serves a large urban center. Estimates of community drug consumption of other opioids indicated that the use of oxycodone was greater in the smaller city relative to the larger city. This is probably due to the older demographic in the smaller city, but it cannot be ruled out that oxycodone is a major drug of abuse in this community. Ketamine was also detected in larger urban centers, which indicates that there is a problem with abuse of this veterinary anesthetic. The analysis of both untreated and treated wastewater indicated that removals of prescription opioids were generally negative; probably as a result of de-conjugation of these compounds as a result of microbial activity during the treatment process to release the parent compound and further investigation using improved sampling strategies is required to better assess of the removal of these drugs during wastewater treatment. While monitoring with the POCIS passive sampler was a convenient method for monitoring for these drugs, problems encountered while estimating concentrations in untreated wastewater limited the use of the data for calculating community drug consumption.

Supplementary data to this article can be found online at http://dx. doi.org/10.1016/j.scitotenv.2013.11.094.

\section{Acknowledgments}

We gratefully acknowledge the assistance of the staff of the wastewater treatment plants for collecting the samples. This work was supported by the Natural Sciences and Engineering Research Council (NSERC) of Canada through Discovery grants to Dr. Yargeau and Dr. Metcalfe, and a Strategic Grant to Dr Yargeau (PI) and colleagues.

\section{References}

Alvarez DA, Huckins JN, Petty JD, Jones-Lepp T, Stuer-Lauridsen F, Getting DT, et al. Chapter 8 tool for monitoring hydrophilic contaminants in water: polar organic chemical integrative sampler (POCIS). In: Greenwood R, G.M., Vrana B, editors. Comprehensive Analytical Chemistry, vol. 48. Elsevier; 2007. p. 171-97.

Boleda MR, Galceran MT, Ventura F. Trace determination of cannabinoids and opiates in wastewater and surface waters by ultra-performance liquid chromatographytandem mass spectrometry. J Chromatogr A 2007;1175:38-48.

Boleda MR, Galceran MT, Ventura F. Monitoring of opiates, cannabinoids and their metabolites in wastewater, surface water and finished water in Catalonia, Spain. Water Res 2009;43:1126-36.

Bones J, Thomas KV, Paull B. Using environmental analytical data to estimate levels of community consumption of illicit drugs and abused pharmaceuticals. J Environ Monit 2007;9.

Carlson J, Challis J, Hanson M, Wong C. Stability of pharmaceuticals and other polar organic compounds stored on polar organic chemical integrative samplers and solid-phase extraction cartridges. Environ Toxicol Chem 2013;32:337-44.

Castiglioni S, Zuccato E, Crisci E, Chiabrando C, Fanelli R, Bagnati R. Identification and measurement of illicit drugs and their metabolites in urban wastewater by liquid cromatography-tandem mass spectrometry. Anal Chem 2006;78:8421-9.

City-Data.com. Population, house counts and age characteristics. City-Data.com; 2012.

Dotson J, Ackerman D, West L. Ketamine abuse. J Drug Issues 1995;25:751-8.

Harman C, Boyum O, Thomas KV, Grung M. Small but different effect of fouling on the uptake rates of semipermeable membrane devices and polar organic chemical integrative samplers. Environ Toxicol Chem 2009;28:2324-32.

Harman C, Reid M, Thomas KV. In situ calibration of a passive sampling device for selected illicit drugs and their metabolites in wastewater, and subsequent year-long assessment of community drug usage. Environ Sci Technol 2011;45:5676-82.

Jones-Lepp TL, Alvarez DA, Petty JD, Huckins JN. Polar organic chemical integrative sampling and liquid chromatography-electrospray/ion-trap mass spectrometry for assessing selected prescription and illicit drugs in treated sewage effluents. Arch Environ Contam Toxicol 2004;47:427-39.

Kasprzyk-Hordern B, Dinsdale RM, Guwy AJ. The removal of pharmaceuticals, personal care products, endocrine disruptors and illicit drugs during wastewater treatment and its impact on the quality of receiving waters. Water Res 2009;43:363-80.

Lai FY, Ort C, Gartner C, Carter S, Prichard J, Kirkbride P, et al. Refining the estimation of illicit drug consumptions from wastewater analysis: co-analysis of prescription pharmaceuticals and uncertainty assessment. Water Res 2011;45:4437-48.

Lee JS, Yang WK, Han EY, Lee SY, Park YH, Lim MA, et al. Monitoring precursor chemicals of methamphetamine through enantiomer profiling. Forensic Sci Int 2007;173: 68-72.

Li H, Helm PA, Metcalfe CD. Sampling in the Great Lakes for pharmaceuticals, personal care products, and endocrine-disrupting substances using the passive polar organic chemical integrative sampler. Environ Toxicol Chem 2010a;29:751-62.

Li H, Vermeirssen ELM, Helm PA, Metcalfe C. A controlled field evaluation of water flow rate effects on sampling polar organic compounds using polar organic integrative samplers (POCIS). Environ Toxicol Chem 2010b;29:2461-9.

Li H, Helm PA, Paterson G, Metcalfe C. The effects of dissolved organic matter and $\mathrm{pH}$ on sampling rates for polar organic chemical integrative samplers (POCIS). Chemosphere 2011;83:271-80.

MacLeod SL, McClure EL, Wong CS. Laboratory calibration and field deployment of the Polar organic chemical integrative sampler for pharmaceuticals and personal care products in wastewater and surface water. Environ Toxicol Chem 2007;26:2517-29.

Majewsky M, Gallé T, Bayerle M, Goel R, Fischer K, Vanrolleghem PA. Xenobiotic removal efficiencies in wastewater treatment plants: residence time distributions as a guiding principle for sampling strategies. Water Res 2011;45:6152-62.

McCaughan J, Carlson R, Falck R, Siegal H. From "Candy Kids" to "Chemi-Kids": a typology of young adults who attend raves in the midwestern United States. Subst Use Misuse 2005;40:1503-23. 
Metcalfe C, Tindale K, Li H, Rodayan A, Yargeau V. Illicit drugs in Canadian municipal wastewater and estimates of community drug use. Environ Pollut 2010;158: 3179-85.

Morin N, Miège C, Coquery M, Randon J. Chemical calibration, performance, validation and applications of the polar organic chemical integrative sampler (POCIS) in aquatic environments. TrAC Trends Anal Chem 2012;36:144-75.

Ort C, Lawrence MG, Rieckermann J, Joss A. Sampling for pharmaceuticals and personal care products (PPCPs) and illicit drugs in wastewater systems: are your conclusions valid? A critical review. Environ Sci Technol 2010;44:6024-35.

Paglia-Boak A, Adlaf EM, Mann RE, Drug use among Ontario students. 1977-2011 OSDUHS-report research document series number 32. Ontario, Canada: Centre for Addiction and Mental Health Toronto; 2011.

Postigo C, Lopez de Alda MJ, Barcelo D. Fully automated determination in the low nanogram per liter level of different classes of drugs of abuse in sewage water by on-line solid-phase extraction-liquid chromatography-electrospray-tandem mass spectrometry. Anal Chem 2008;80:3123-34.

Postigo C, Lopez de Alda MJ, Barcelo D. Drugs of abuse and their metabolites in the Ebro River basin: occurrence in sewage and surface water, sewage treatment plants removal efficiency, and collective drug usage estimation. Environ Int 2010;36:75-84.

Postigo C, de Alda ML, Barcelo D. Evaluation of drugs of abuse use and trends in a prison through wastewater analysis. Environ Int 2011;37:49-55.

Radbruch L, Grond S, Lehmann KA. A risk-benefit assessment of tramadol in the management of pain. Drug Saf 1996;15:8-29.
Ternes T. Occurrence of drugs in German sewage treatment plants and rivers. Water Res 1998;32:3245-60.

Terzic S, Senta I, Ahel M. Illicit drugs in wastewater of the city of Zagreb (Croatia) estimation of drug abuse in a transition country. Environ Pollut 2010;158:2686-93.

Trescot AM, Datta S, Lee M, Hansen H. Opiod pharmacology. Pain Physician 2008: S133-53. (Opiod Special Issue 2008).

van der Aa NGFM, Dijkman E, Bijlma L, Emke E, van de Ven BM, van Nuijs ALN, et al. Drugs of abuse and tranquilizers in Dutch surface waters, drinking water and wastewater: results of screening monitoring 2009. 90National Institute for Public Health and the Environment; 2010.

Van Havere T, Vanderplasschen W, Lammertyn J, Broekaert E, Bellis M. Drug use and nightlife: more than just dance music. Subst Abuse Treat Prev Policy 2011;6.

van Nuijs ALN, Castiglioni S, Tarcomnicu I, Postigo C, de Alda ML, Neels H, et al. Illicit drug consumption estimations derived from wastewater analysis: a critical review. Sci Total Environ 2011a;409:3564-77.

van Nuijs ALN, Mougel J-F, Tarcomnicu I, Bervoets L, Blust R, Jorens PG, et al. Sewage epidemiology - a real-time approach to estimate the consumption of illicit drugs in Brussels, Belgium. Environ Int 2011b;37:612-21.

Yacoubian GJ, C.B., Harding CA, Loftus E. It's a rave new world: estimating the prevalence and perceived harm of ecstasy and other drug use among club rave attendees. J Drug Educ 2003;33:187-96.

Zuccato E, Chiabrando C, Castiglioni S, Bagnati R, Fanelli R. Estimating community drug abuse by wastewater analysis. Environ Health Perspect 2008;116. 